\title{
Editorial: 25 years of the analysis of stability of the replica-symmetric solution of the Sherrington-Kirkpatrick model of a spin glass
}

This issue of the Braz. J. Phys. brings an article of Jairo R. L. de Almeida, who was a pioneer in the investigation of spin glasses in Brazil. It is fitting, therefore, to recall to the readers that this year marks the silver jubilee of the influential paper "Stability of the Sherrington-Kirkpatrick solution of a spin glass model" by Jairo R. L. de Almeida and David J. Thouless (J. Phys. A11, 983, 1978). Three years before this publication, David Sherrington and Scott Kirkpatrick had introduced an infinite-ranged Ising spin glass, which they claimed to have solved exactly using the novel replica method. They were forced to recognize, however, that the (presumably) exact solution suffered from a grave inconsistency, for the entropy became negative at low temperatures. The obvious culprit was the replica method. Either the interchange of limits (the thermodynamic limit, $N \rightarrow \infty$, and the number of replicas limit, $n \rightarrow 0$ ) or the analytic continuation from an integer $n \geq 1$ number of replicas to $n \rightarrow 0$ were the main suspected problems. Surprisingly, de Almeida and Thouless showed that the fault was not the replica method, but the symmetric saddle-point solution postulated by Sherrington and Kirkpatrick. In a now standard calculation, they diagonalized a Hessian matrix exactly, for integer $n$, and analytically continued the eigenvalues to $n=0$. One of the eigenvalues becomes negative below a line, known as the "AlmeidaThouless line", which indicates that the symmetric solution is unstable at low temperatures. The insight provided by this work enabled Giorgio Parisi a year later, in a brilliant guesswork, to infer a highly nontrivial saddle-point solution in the replica space. Parisi's replica-symmetry-breaking solution has stood all of the tests of stability carried out to date, and it is generally accepted as the correct solution for the spin-glass phase of the (mean-field) Sherrington-Kirkpatrick model. Indeed, in a very recent paper, the mathematician Michel Talagrand gives a proof that Parisi's functional order parameter leads to the correct limiting free energy of this model system.

The relevance of the work by de Almeida and Thouless can be gauged by more than a thousand citations of their paper. Incidentally, a casual search in the ISI database for this paper may give a deceptively small number of citations. This unfortunate situation stems from the alternative ways, Almeida or de Almeida, that have been used to cite the author. Apart from the historical role as a herald of Parisi's solution, the method pioneered by de Almeida and Thouless remains an essential tool for analyzing the stability of the replica-symmetric solutions of a large class of spin-glass and neural-network models. In this same issue of the Braz. J. Phys, we publish an article with a complete analysis of stability of the replica-symmetric solution of a two-sublattice version of the Sherrington-Kirkpatrick model (which is meant to represent a phase transition from an antiferromagnetic to a spin-glass phase). Although the long-ranged, mean-field, Sherrington-Kirkpatrick model may be far from the more realistic, short-ranged, Edwards-Anderson spin-glass model for magnetic systems, the seminal paper of de Almeida and Thouless will certainly be of enduring importance in the analysis of different sorts of optimization problems related to the broad domains of complex systems.

Carlos S. O. Yokoi*, Francisco A. da Costa ${ }^{\dagger}$, and Silvio R. A. Salinas*

${ }^{*}$ Instituto de Física, Universidade de São Paulo

†Departamento de Física Teórica e Experimental, Universidade Federal do Rio Grande do Norte

December, 2003 